\title{
Fast detection of SARS-CoV-2 RNA via the integration of plasmonic thermocycling and fluorescence detection in a portable device
}

\author{
Jiyong Cheong ${ }^{1,2,7}$, Hojeong Yu ${ }^{1,2,3,4,7}$, Chang Yeol Lee ${ }^{1,2}$, Jung-uk Lee ${ }^{1,5}$, Hyun-Jung Choi ${ }^{6}$,

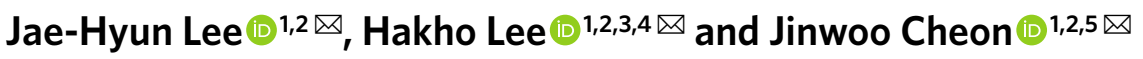

The diagnosis of severe acute respiratory syndrome 2 (SARS-CoV-2) infection by quantitative PCR with reverse transcription (RT-qPCR) typically involves bulky instrumentation in centralized laboratories and an assay time of 1-2 h. Here, we show that SARS-CoV-2 RNA can be detected in $17 \mathrm{~min}$ via a portable device integrating reverse transcription, fast thermocycling (via plasmonic heating through magneto-plasmonic nanoparticles) and in situ fluorescence detection following magnetic clearance of the nanoparticles. The device correctly classified all nasopharyngeal, oropharyngeal and sputum samples from 75 patients with COVID-19 and 75 healthy controls, with good concordance in fluorescence intensity with standard RT-qPCR (Pearson coefficients $>0.7$ for the N1, N2 and RPP30 genes). Fast, portable and automated nucleic acid detection should facilitate testing at the point of care.

$\mathrm{D}$ eveloping fast and reliable diagnostic tools is critical during the current coronavirus disease 2019 (COVID-19) pandemic, as diagnostic capacity at scale becomes critical to containing outbreaks and to reducing fatality rates ${ }^{1-7}$. In the absence of effective cures or vaccines for the disease, identifying as many infected individuals as possible (both symptomatic and asymptomatic) and then isolating them is the most effective way to prevent disease transmis$\operatorname{sion}^{5,6,8}$. Administering on-site and rapid tests is ideal for prompt patient triaging and contact tracing. Unfortunately, diagnostic tools with such capabilities are not readily available. Immunogenic lateral-flow assays are fast, small and cost effective, but are unsuitable for viral detection during the early disease phase ${ }^{9-11}$. Nucleic acid amplification tests based on PCR are the gold standard for COVID-19 confirmation, owing to their high analytical accuracy $(\sim 99 \%)^{12,13}$. A caveat, however, is that most PCR tests are carried out in centralized laboratories, which often incur logistic overheads (such as sample transfer and protection from degradation) and long turnaround times $(2-3 \mathrm{~d})^{14-16}$. The deployment of conventional PCR to point-of-care (POC) settings is also limited by lengthy assay times $(\sim 1-2 \mathrm{~h})$ and the need for bulky instrumentation. New isothermal nucleic acid amplification technologies may shorten assay times, but they are less established than conventional PCR (because of supply chain issues and the need for clinical validation) and may have inferior accuracy ${ }^{17,18}$.

Rapid and portable thermocycling technology aims to bring nucleic acid amplification tests to POC settings. Thermoplasmonics is a promising method for fast heating ${ }^{19-25}$. It exploits the light-to-heat conversion mediated by plasmonic substrates ${ }^{26-29}$. This concept has been adopted for PCR thermocycling, in the form of a planar plasmonic substrate in a fluidic device ${ }^{20,30,31}$ or metallic nanoparticles in suspension $^{22,32,33}$. Both methods have shown fast nucleic acid ampli- fication, but challenges remain: nucleic acids after amplification are difficult to detect because plasmonic substrates would interfere with fluorescence reporters ${ }^{34,35}$. Improving the detection sensitivity often entails additional steps involving the use of external equipment (in particular, centrifugation (for particle removal), gel electrophoresis (for separate signal detection) or a microscope system) (Supplementary Table 1). These requirements would lengthen the total assay time, overshadowing the advantage of fast thermocycling, and would also complicate the workflow.

Here, we report the development of a fast one-pot PCR with reverse transcription (RT-PCR) technology for COVID-19 diagnostics. We named it nanoPCR. The system seamlessly integrates plasmonic thermocycling with fluorescent signal detection in a single device. A key concept is the use of dual-functional magneto-plasmonic nanoparticles (MPNs) for PCR applications. We noticed that most of the plasmonic effect is confined near the surface of plasmonic nanoparticles. By encasing a magnetic core with a plasmonic gold (Au) shell, we could thus achieve: (1) efficient plasmonic heating comparable to that by solid Au nanoparticles; and (2) rapid nanoparticle separation with external magnetic fields to perform in situ signal detection. Exploiting these advantages, we advanced a compact nanoPCR system that automatically executes reverse transcription, rapid PCR amplification and fluorescence detection with a single button press. The current prototype concurrently measures three samples within $17 \mathrm{~min}$. The limit of detection is 3.2 gene copies per $\mu \mathrm{l}$, which is comparable to those by benchtop PCR equipment. We further applied nanoPCR to test clinical samples (75 patients with COVID-19 and 75 controls). The nanoPCR device rapidly detected three gene targets (N1, N2 and RPP30) and achieved excellent diagnostic accuracy $(>99 \%)$.

${ }^{1}$ Center for Nanomedicine, Institute for Basic Science (IBS), Seoul, Republic of Korea. ${ }^{2}$ Graduate Program of Nano Biomedical Engineering (NanoBME), Advanced Science Institute, Yonsei University, Seoul, Republic of Korea. ${ }^{3}$ Center for Systems Biology, Massachusetts General Hospital Research Institute, Boston, MA, USA. ${ }^{4}$ Department of Radiology, Massachusetts General Hospital, Harvard Medical School, Boston, MA, USA. ${ }^{5}$ Department of Chemistry, Yonsei University, Seoul, Republic of Korea. ${ }^{6}$ Department of Laboratory Medicine, Chonnam National University Hospital, Chonnam National University Medical School, Gwangju, Republic of Korea. ${ }^{7} T h e s e$ authors contributed equally: Jiyong Cheong, Hojeong Yu. ${ }^{凶}$ e-mail: jhyun_lee@yonsei.ac.kr;

hlee@mgh.harvard.edu; jcheon@yonsei.ac.kr 
a

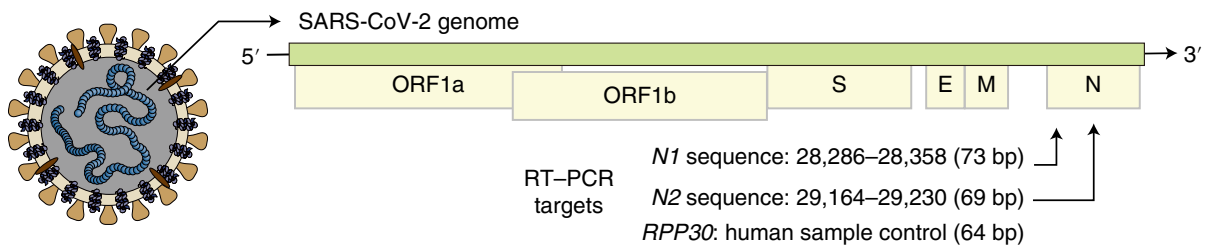

b

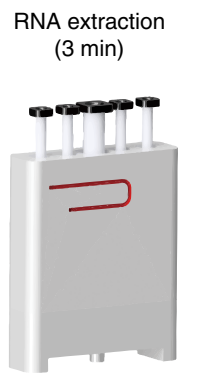

Disposable preparation kit
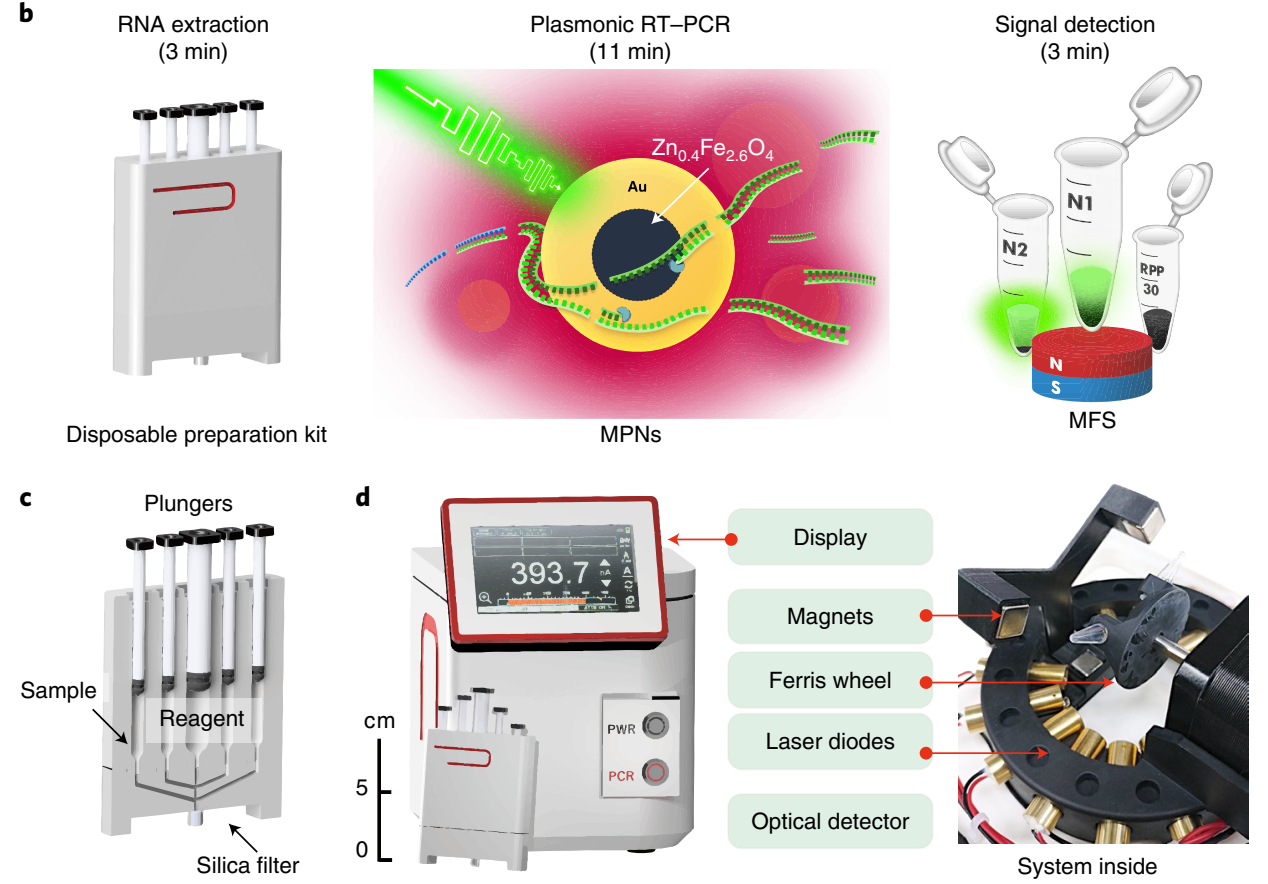

e

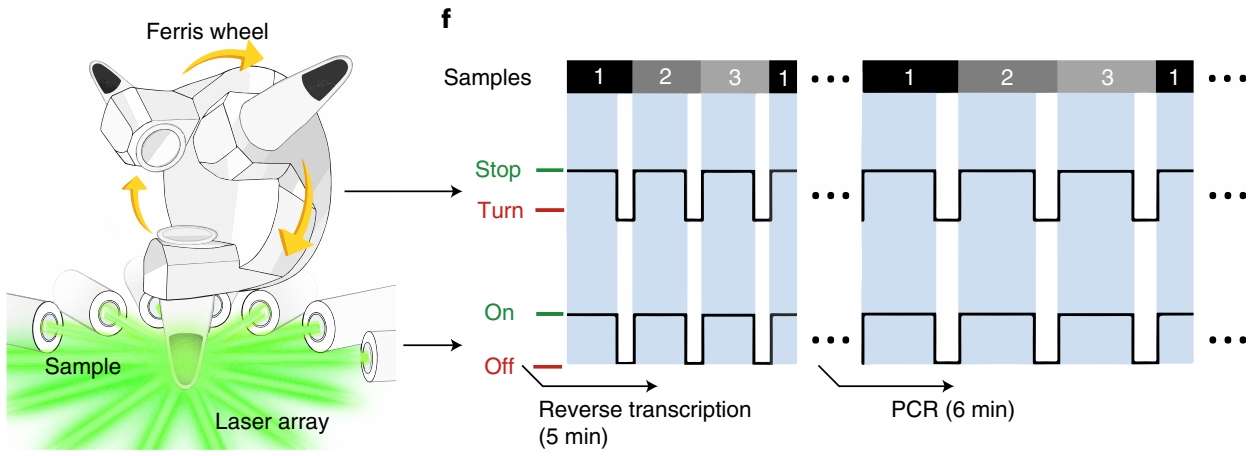

Fig. 1 | NanoPCR assay schematics for COVID-19 diagnostics. a, Target RNA regions for nanoPCR tests. The N1 and N2 genes are for SARS-CoV-2 detection, whereas the RPP30 gene serves as a control for human sample confirmation. ORF, open reading frame; $\mathrm{S}$, spike; $\mathrm{E}$, envelope; $M$, membrane; $N$, nucleocapsid. $\mathbf{b}$. High-speed nanoPCR diagnostic flow for SARS-CoV-2 detection: (1) 3 min of RNA extraction using a disposable RNA preparation kit; (2) 11 min of RT-PCR by magneto-plasmonic thermocycling; and (3) 3 min of detection and diagnosis via MFS by application of an external magnetic field for MPN removal. c, A disposable RNA extraction kit with preloaded buffers and a simple plunge system. d, A compact nanoPCR instrument for POC application. The system houses a light source for plasmonic heating, a Ferris wheel for sample mounting, MFS magnets and optics for fluorescence detection. e, Circular array of the low-powered laser diodes and synchronized Ferris wheel for thermocycling of multiple samples. $\mathbf{f}$, Syncing of laser illumination with Ferris wheel rotation to enable the processing of multiple samples without compromising the total assay time.

\section{Results and discussion}

We used nucleic acid targets recommended by the US Center for Disease Control and Prevention (Fig. 1a) ${ }^{36}:$ N1 and N2 genes (for severe acute respiratory syndrome 2 (SARS-CoV-2) virus detection) and the human RPP30 gene (for human sample confirmation). Figure $1 \mathrm{~b}$ shows the nanoPCR schematic for 17-min COVID-19 diagnostics (see Supplementary Video 1 for the whole workflow). The assay starts with extracting total RNAs from suspected patient samples (for example, nasopharyngeal/oropharyngeal swabs or sputum). Extracted RNA samples are then mounted inside a nanoPCR device, and the remaining assay steps are carried out automatically: (1) thermoplasmonic RT-PCR (11 min); (2) magnetic fluorescence switch (MFS) to remove MPNs from samples using an external magnetic field (3 min); and (3) fluorescence signal detection directly from tubes $(<20 \mathrm{~s})$. Overall, the nanoPCR offers practical advantages for on-site molecular diagnostics. The dual-functional 
MPNs enable both rapid nucleic acid amplification (via plasmonic heating) and the detection of fluorescent signal (via MPN clearing) in a one-pot assay format.

To facilitate sample preparation at POC settings, we designed a disposable RNA preparation kit (Fig. 1c and Supplementary Fig. 1). The kit has multiple chambers preloaded with RNA extraction reagents (see Methods for details) and a silica filter to capture RNA (that is, solid-phase extraction). After loading a test sample $(20 \mu \mathrm{l})$, we sequentially actuated plungers to perform virus lysis, RNA capture and elution to PCR tubes. When tested with human coronavirus NL63, the kit achieved extraction yields comparable to those achieved by a conventional centrifugation method (Supplementary Fig. 2); however, the kit operation was equipment free and complete within 3 min.

We implemented a compact nanoPCR system, integrating key components for plasmonic RT-PCR. Specifically, the system had a light source for plasmonic heating, a rotating sample holder (Ferris wheel style), movable magnets and a fluorescent optical setup (Fig. 1d). A microcontroller coordinated the operation of individual modules: with a single button push, the system automatically executed plasmonic RT-PCR, MFS and fluorescence detection and displayed the results on an embedded screen (Supplementary Video 1). This self-contained system had a small form factor $\left(15 \times 15 \times 18.5 \mathrm{~cm}^{3}\right)$ and weighed $\sim 3 \mathrm{~kg}$ (Fig. 1d).

As the plasmonic light source, we arranged weak-powered $(80 \mathrm{~mW})$ laser diodes in a circular array (Fig. 1e and Supplementary Fig. 3), which allowed for more even illumination on samples than using a single high-power $(\sim 1 \mathrm{~W})$ laser. For the sample mounting, we adopted a Ferris wheel scheme to achieve higher throughput. The wheel rotation sequentially brought a sample under light illumination while other samples were air cooled. By syncing the wheel rotation and the illumination schedule (Fig. 1f), we could perform PCR reactions in multiple samples using an existing light source. The method thereby provided an economical way to scale up (see Supplementary Note 2 for details). The current POC prototype was designed for three samples. We also constructed a prototype that could process nine samples (Supplementary Fig. 4), which had the throughput of processing roughly 36 samples per hour.

The essential nanoPCR reagent was the dual-functional MPNs for plasmonic heating and magnetic clearing. The particle had a 16-nm magnetic core $\left(\mathrm{Zn}_{0.4} \mathrm{Fe}_{2.6} \mathrm{O}_{4}\right)$ enclosed by a 12-nm-thick $\mathrm{Au}$ shell (Fig. 2a, left) ${ }^{37}$. Elemental mapping with energy dispersive $\mathrm{X}$-ray spectroscopy (EDS) confirmed the MPNs' core-shell nature (Fig. 2a, right). We further coated the particles with phosphinesulfonate ligands, which stabilized MPNs by imparting negative surface charges. The hydrodynamic size of MPNs measured with dynamic light scattering (DLS) was $\sim 50 \mathrm{~nm}$ without aggregation and they maintained excellent colloidal stability for 1 year without a change in size (Supplementary Fig. 5).

We characterized the MPNs' optical properties, measuring plasmonic responses and simulating electric fields. With the 12-nm-thick Au shell, the MPNs showed plasmon resonance at a wavelength $(\lambda)$ of $535 \mathrm{~nm}$, which matched with the peak wavelength $(532 \mathrm{~nm})$ of the laser diode used in the system (Fig. 2b). The electric field map under plasmonic resonance, simulated by the boundary element method (see Methods), indicated that the field enhancement was particularly confined to both the inner and outer surfaces of the Au shell (Fig. 2c,d). In addition, the electric field enhancement $\left(E / E_{0}\right)$ and cross-section absorption $\left(\sigma_{\mathrm{abs}}\right)$ of a single MPN were similar to those of a solid 40-nm Au nanoparticle (Supplementary Fig. 6). These results supported that the core-shell structure could maintain the desired surface plasmonic properties while providing additional functionality through the core.

Magnetic measurements confirmed that the MPNs were superparamagnetic, with a magnetic moment of $m=7.52 \times 10^{-19} \mathrm{~A} \mathrm{~m}^{2}$ per particle (Fig. 2e). Under the magnetic field generated by an external
$\mathrm{NdFeB}$ magnet, MPNs clustered through dipole-dipole interactions, which increased the overall hydrodynamic size (from 50 to $530 \mathrm{~nm}$ ) (Fig. 2f,g). The magnetic moment of clustered MPNs was estimated to be much higher $\left(8.96 \times 10^{-16} \mathrm{~A} \mathrm{~m}^{2}\right)$-approximately 1,200 times higher than a single MPN (see Supplementary Note 1). This feature enabled us to quickly clear MPNs from solution, facilitating the interference-free fluorescent detection.

We used MPNs as a volumetric heating source in the plasmonic RT-PCR. Shining light on the MPN-containing solution quickly heated the entire sample (Fig. 3a). The heating effect was dominant only when the incident wavelength matched to the MPNs' plasmonic resonance (Fig. 3b), confirming the underlying thermoplasmonic mechanism. We further developed an analytical model to estimate the temperature increase in the MPN solution. Approximating a single MPN as a spherical heater, the temperature profile around the particle is given as $\Delta T_{\mathrm{MPN}}=\left(\sigma_{\mathrm{abs}} I\right) /\left(4 \pi R k_{\mathrm{s}}\right)$, where $k_{\mathrm{s}}$ is the thermal conductivity of the solution, $I$ is the irradiance of the illumination and $R$ is the particle radius ${ }^{38}$. Under our experimental conditions $\left(\sigma_{\mathrm{abs}}=2.5 \times 10^{3} \mathrm{~nm}^{2}, R=20 \mathrm{~nm}, k_{\mathrm{s}}=0.6 \mathrm{~W} \mathrm{~m}^{-1} \mathrm{~K}^{-1}\right.$ for water and $\left.I=\sim 0.1 \mathrm{~W} \mathrm{~mm}^{-2}\right)$, the overall temperature increase of the solution is estimated as $\Delta T=\Delta T_{\mathrm{MPN}}(R / \delta) N^{2 / 3}$, where $N$ is the total number of MPNs and $\delta$ is the inter-particle distance ${ }^{39}$. With $N$ particles suspended in volume $V, \delta=\sim 0.55(V / N)^{1 / 3}$, leading to $\Delta T=2 \Delta T_{\mathrm{MPN}}(N / V) V^{2 / 3}$. Therefore, a linear increase in $\Delta T$ with MPN concentration ([MPN]) is expected (Fig. 3c, dotted lines), which agrees well with the experimental observations (Fig. 3c, circles). The heating efficiency, however, deteriorated when $[\mathrm{MPN}] \gtrsim 3 \times 10^{11} \mathrm{ml}^{-1}$; this could be attributed to shifts in plasmonic resonance caused by inter-particle coupling ${ }^{40}$. To maximize the heating efficiency, we set $[\mathrm{MPN}]=2.6 \times 10^{11} \mathrm{ml}^{-1}$ for the rest of the assay.

Applying MPN plasmonic heating, we could achieve rapid thermocycling ( 58 to 90 to $58^{\circ} \mathrm{C}$ ) at a rate of $8.91 \mathrm{~s}$ per cycle (Fig. $3 \mathrm{~d}$ ). The precision at target temperatures was excellent, with the coefficient of variations $<1.64 \%$ (Fig. 3e). Note that we relied on the convective heat transfer to air for the sample cooling. The observed cooling time from 90 to $58^{\circ} \mathrm{C}$ was $\sim 6.5 \mathrm{~s}$, which also agreed with the numerical modelling (Supplementary Fig. 7). Such fast cooling was possible mainly because the sample volume was small $(10 \mu \mathrm{l})$. For COVID-19 detection, we tuned the Ferris wheel for three-sample measurement (Fig. 3f), with each sample targeting one of three genes for COVID-19 diagnostics (that is, N1, N2 and RPP30). By synching the wheel rotation with the heating cycle $(2.43 \mathrm{~s}$ under light; $6.48 \mathrm{~s}$ with air cooling), we could continuously process three samples using a light source designed for a single PCR tube (Fig. $3 \mathrm{~g}$ and Supplementary Fig. 8).

Next, we optimized the entire operation for SARS-CoV-2 gene detection (Fig. 4a). The process required maintaining a constant temperature for reverse transcription. We controlled the effective light power by modulating the on/off duty cycle, achieving the target temperature $\left(42^{\circ} \mathrm{C}\right)$ with small variations $\left(<1^{\circ} \mathrm{C}\right.$; Fig. 4 a right $)$. Testing with N1, N2 and RPP30 target genes, we confirmed that the 5 -min reverse transcription produced sufficient numbers of complementary DNAs (see Methods and Supplementary Fig. 9). Following the reverse transcription reaction, we executed the PCR amplification (40 cycles per tube; $6 \mathrm{~min}$ ). Gel electrophoresis on PCR products by nanoPCR ( $6 \mathrm{~min}$ ) and by conventional benchtop instrument $(2 \mathrm{~h})$ showed matching bands (Fig. 4b), confirming comparable performance between two systems. With 5-min conventional PCR, no band was detected (Supplementary Fig. 10). We further assessed the purity of the PCR products by measuring ultraviolet absorbance ratios $\left(A_{260} / A_{230}\right.$ and $\left.A_{260} / A_{280}\right)$, where $A_{230}, A_{260}$ and $A_{280}$ are the ultraviolet absorbance at 230,260 and $280 \mathrm{~nm}$, respectively (Supplementary Fig. 11). The measured ratios were $A_{260} / A_{230}=\sim 2.2$ and $A_{260} / A_{280}=\sim 1.8 . A_{260} / A_{230}$ was higher than $A_{260} / A_{280}$, indicating that nanoPCR produced high-quality PCR products. 
a

Plasmonic resonance
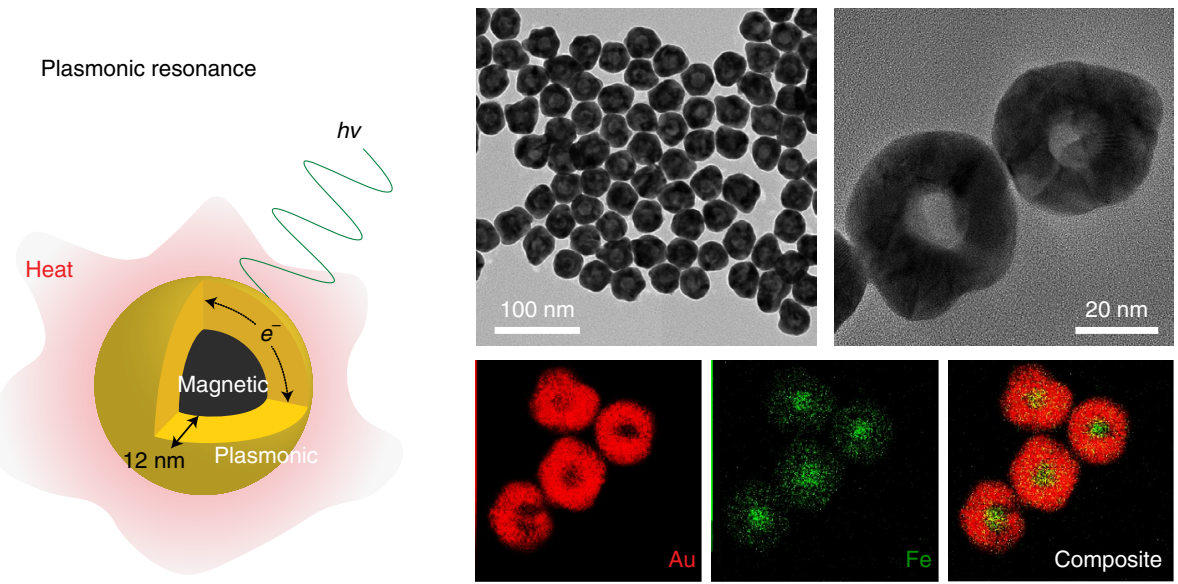

b
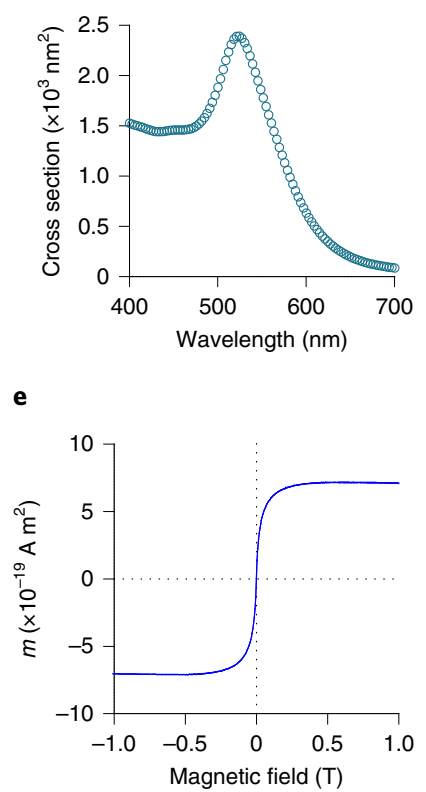

c
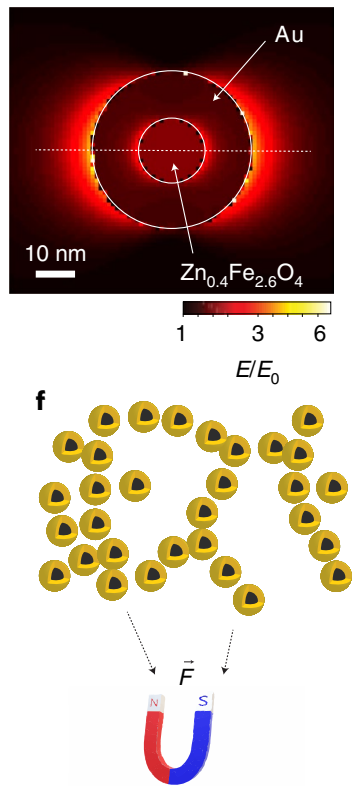

d

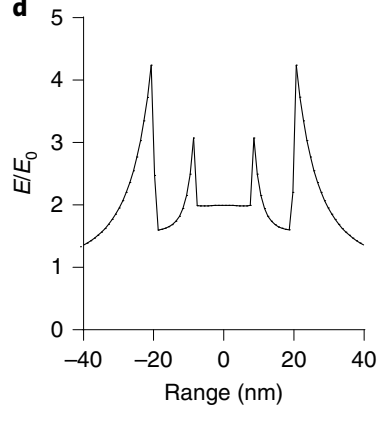

g

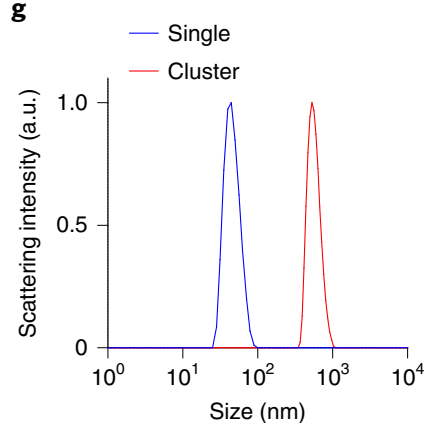

Fig. 2 | MPNs. a, TEM images of MPNs with a core-shell ( $\mathrm{Zn}_{0.4} \mathrm{Fe}_{2.6} \mathrm{O}_{4}$-Au) structure. Left: a 16-nm magnetic core was encased by a $12-\mathrm{nm}$ Au shell. Right: elemental mapping of Au and Fe showed an area-specific distribution of the core and Au shell structure. $\mathbf{b}$, Ultraviolet-visible cross-section absorption spectrum of an MPN measured with the integrating sphere. At a shell thickness of $12 \mathrm{~nm}$, the highest absorbance was measured at $535 \mathrm{~nm}$. c, Simulation of the electric field at $535 \mathrm{~nm}$ illumination. An intense electric field was confined to the Au surface. d, Linear profile of the maximum electric field enhancement factor $\left(E / E_{0}\right.$ ) (dotted line in $\mathbf{c}$ ). e, Estimated magnetization curve of a single MPN, as measured using a vibrating sample magnetometer. MPNs are superparamagnetic at room temperature $\left(20^{\circ} \mathrm{C}\right)$. f, The clustered form of MPN was realized by dipolar interaction under magnetic field application (100 T m ${ }^{-1}$ ). $\mathbf{g}$, Hydrodynamic sizes of MPNs before and after clustering, as measured by DLS. The size increased from 50 to 530 nm.

For fluorescence signal detection in situ, we applied MFS to clear MPNs in sample tubes. After completing the RT-PCR reaction, the nanoPCR system automatically moved a magnet array to the Ferris wheel (Fig. 4c, left; see Supplementary Video 1) and read out the fluorescence signal using an internal ultraviolet light-emitting diode (LED) and a photodiode (Supplementary Fig. 12). Under the field gradient $\left(100 \mathrm{~T} \mathrm{~m}^{-1}\right)$ created by the magnet, MPNs clustered and sedimented to the tube bottom (see Supplementary Note 1). We observed that MFS was indispensable for reliable measurements. Without MFS, amplicons' signal from fluorescein amidites (FAMs) was barely detectable (Fig. 4c, right), presumably due to the spectral overlap between MPN absorption $\left(\lambda_{\text {peak }}=535 \mathrm{~nm}\right)$ and FAM emission $\left(\lambda_{\mathrm{em}}=517 \mathrm{~nm}\right)$. When tested with $N 1$ amplicons, the fluorescence signal recovered to $\sim 50 \%$ of its saturation level at $3 \mathrm{~min}$ after
MFS (Fig. 4d and Supplementary Fig. 13). Applying this timing, we could reliably detect all three target genes (N1,N2 and RPP30; Fig. 4e). Of note, nanoPCR is inherently limited to end-point measurements, as the assay detects signal only after PCR reaction.

With operational parameters determined, we evaluated the analytical performance of nanoPCR, benchmarking it against conventional RT-PCR. To estimate the limit of detection (LOD), serially diluted samples containing N1, N2 or RPP30 target genes were prepared and subjected to the complete nanoPCR procedures (that is, reverse transcription, 40-cycle PCR and MFS). The LOD was calculated to be 3.2 copies per $\mu \mathrm{l}$ based on the standard formula (threshold $=3 \times$ s.d. of a blank sample; Fig. 4 f), which was comparable to that of conventional RT-PCR $(2.0 \text { copies per } \mu \mathrm{l})^{41,42}$. Furthermore, nanoPCR-based N1 and N2 detection distinguished SARS-CoV-2 

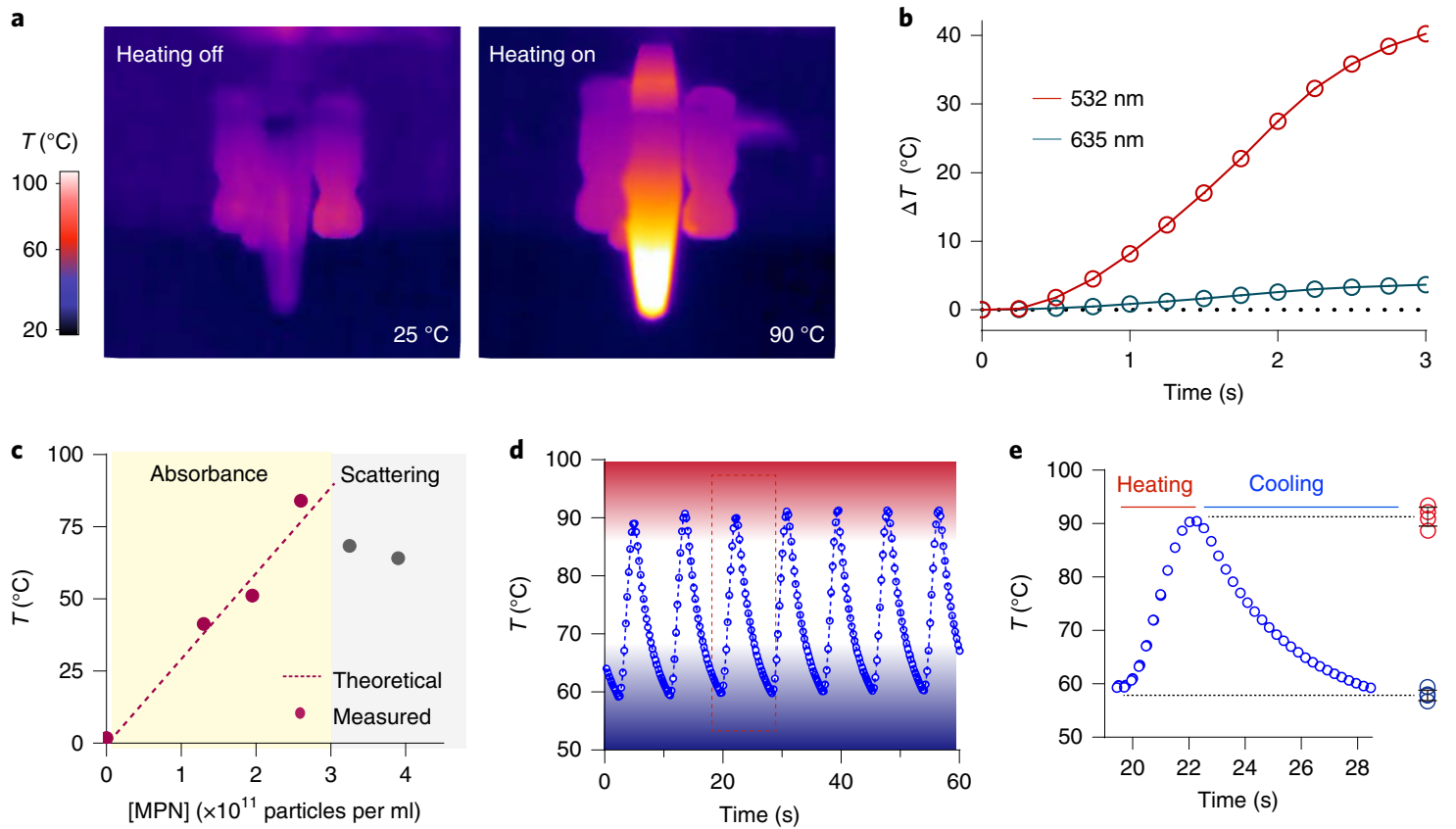

f

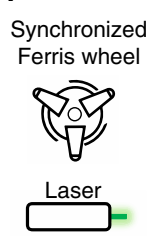

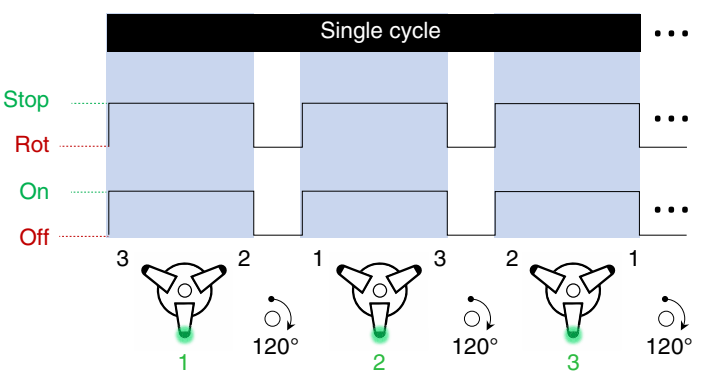

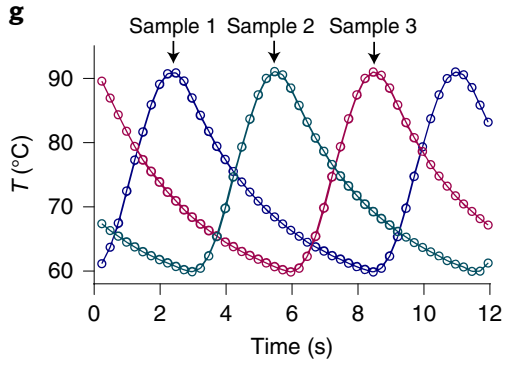

Fig. 3 | MPN-induced plasmonic heating. a, Thermal images of plasmonically heated MPN solution (light source $=1,000 \mathrm{~mW}$ at $\lambda=532 \mathrm{~nm}$; solution volume $=10 \mu \mathrm{l} ; \mathrm{MPN}$ concentration $=2.6 \times 10^{11}$ particles per $\mathrm{ml}$ ). The solution temperature changed from 25 to $90^{\circ} \mathrm{C}$ within a few seconds upon laser illumination. $\mathbf{b}$, Temperature profile of MPN solutions with different wavelengths of light illumination. The laser diode with a wavelength of $532 \mathrm{~nm}$ matched closely to the maximum absorption of MPN; thereby, faster heating was induced. c, Effect of MPN concentration on the temperature profile (dotted line: theoretical estimation; circle: measured values). The maximum temperature increase was observed at a concentration of $2.6 \times 10^{11}$ particles per ml. At higher concentrations, the heating effect degenerated. d, High-speed plasmonic thermocycling profile of MPN solution (seven cycles per min; 58-90 ${ }^{\circ} \mathrm{C}$ ). e, Single thermocycling of MPN solution (red dotted rectangle in $\mathbf{d}$ ). The heating rate was $13.17^{\circ} \mathrm{C} \mathrm{s}^{-1}$ and the cooling rate was $4.94^{\circ} \mathrm{C} \mathrm{s}^{-1}$. The temperature deviations (right) were from five cycles at 58 (blue) and $90^{\circ} \mathrm{C}$ (red). Data are mean \pm s.e.m. The values were $<1 \%$ compared with the target temperature. f, Schematic of the synchronized Ferris wheel system for multi-sample thermocycling. Sample rotation and laser illumination were synced to heat three samples. $\mathbf{g}$, Temperature profiles of three samples on the wheel. Individual heating profiles were interleaved such that the total cycling time remained the same.

from other zoonotic coronaviruses, SARS-CoV and Middle East respiratory syndrome coronavirus, with negligible cross-reactivity (Fig. 4g).

To validate nanoPCR's clinical applicability, we tested clinical samples (that is, nasopharyngeal/oropharyngeal swabs and sputum samples) from cohorts of patients with COVID-19 and control individuals without COVID-19 (see Supplementary Table 2 for patient information). COVID-19 infection status was independently confirmed by the Clinical Diagnostic Laboratory at Chonnam National University Hospital (Republic of Korea). We used the first 100 samples as a discovery cohort (50 patients with COVID-19 and 50 controls) and another 50 samples (25 patients with COVID-19 and 25 controls) as a validation cohort (Fig. 5a). Each patient sample was divided into two. One aliquot was processed using the nanoPCR and the other using a benchtop PCR system. Both assays used the same N1, N2 and RPP30 probes. As a positive reference, we also processed a control sample containing a known amount of reference RNA (synthetic N1 RNA).
Figure $5 b$ shows the results from nanoPCR and quantitative PCR with reverse transcription (RT-qPCR). We compared raw fluorescence intensity $I$ (nanoPCR) with $-\log _{2}[\mathrm{Ct}]$, where $\mathrm{Ct}$ is the cycle cut-off of RT-qPCR; these two quantities would be proportional to target gene concentrations. We observed a good concordance between these two methods. For each gene target, nanoPCR and RT-qPCR showed a strong positive correlation with the Pearson coefficient $(r)$ values of 0.87 (N1), 0.78 (N2) and 0.70 (RPP30).

Next, we assessed the diagnostic accuracy of nanoPCR. As system-independent analytical measures, we defined $F_{N 1}$ and $F_{N 2}$ by normalizing the fluorescence intensities of the target genes (N1 and $N 2$ ) with that of the positive reference (Fig. 5c). The $F_{N 1}$ and $F_{N 2}$ values were significantly higher $(P<0.0001$ for both; two-sided $t$-test $)$ in patients with COVID-19 than in controls (Fig. 5d). We further constructed receiver operating characteristic (ROC) curves for $F_{N 1}$ and $F_{\mathrm{N} 2}$. The diagnostic accuracy was excellent, with an area under the curve of 1 (Fig. 5e) for both $F_{N 1}$ and $F_{N 2}$. From ROC curves, we determined optimal cut-offs, $F_{T H_{-} N 1}=0.15$ for $F_{N 1}$ and $F_{T H \_N 2}=0.05$ 

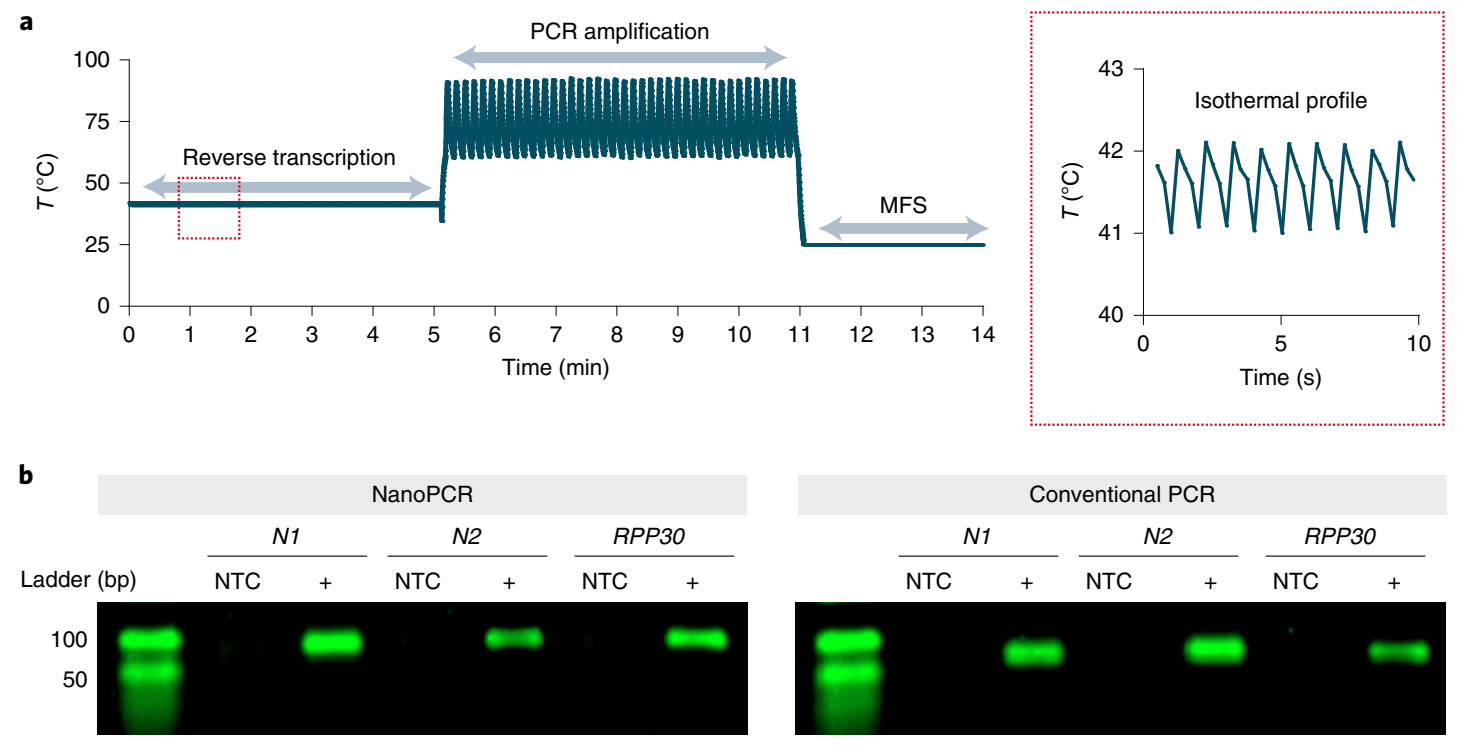

C
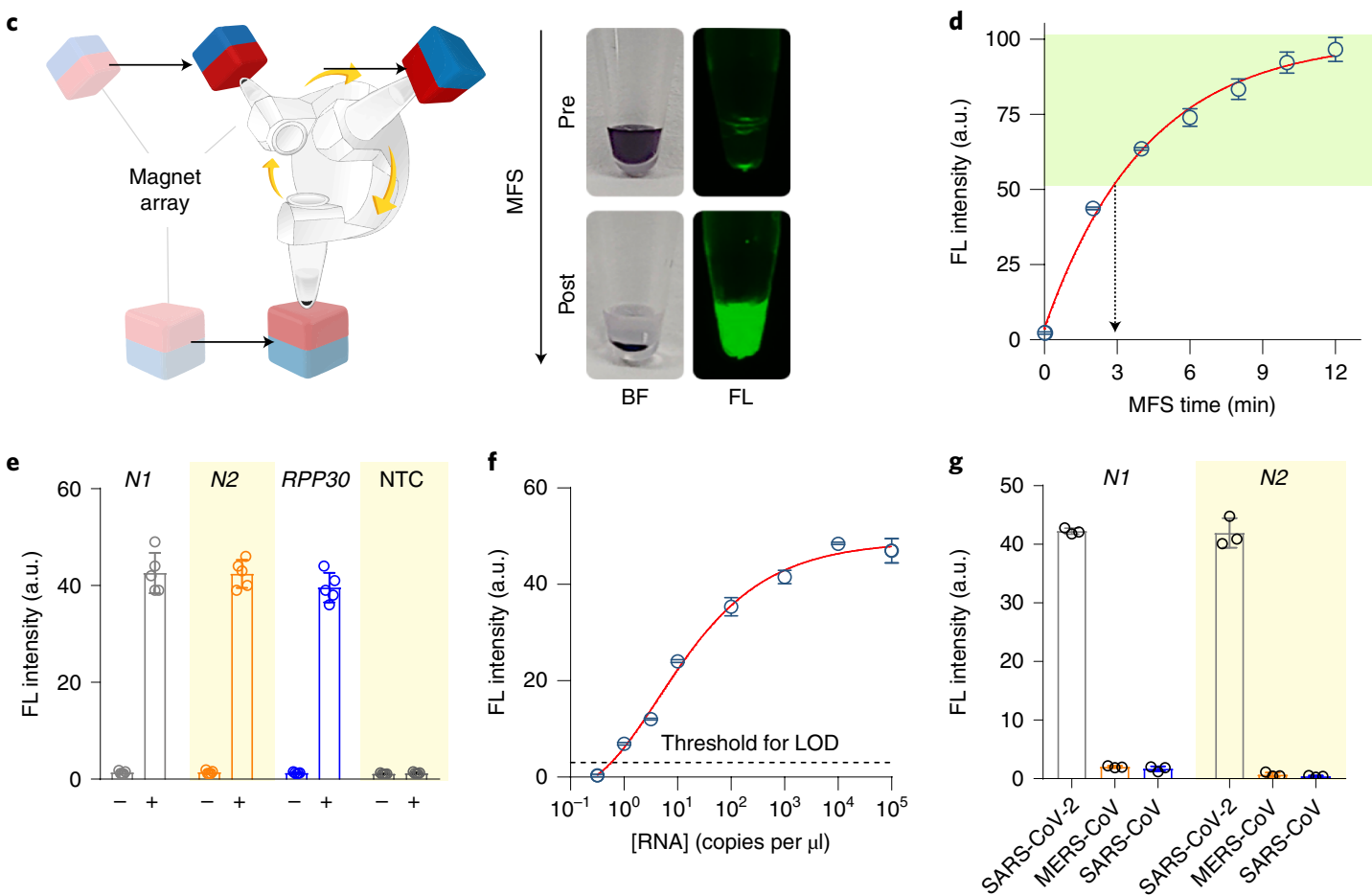

Fig. 4 | NanoPCR detection of SARS-CoV-2 genes. a, Measured temperature profiles of a sample under nanoPCR. Left: $42^{\circ} \mathrm{C}$ incubation for reverse transcription ( $5 \mathrm{~min}$ ), followed by PCR amplification ( 40 cycles; $6 \mathrm{~min}$ ), then signal detection steps ( $3 \mathrm{~min}$ ). The whole RT-PCR process was complete within $11 \mathrm{~min}$. Right: enlarged temperature profile during the isothermal reverse transcription phase. $\mathbf{b}$, Gel electrophoresis images of PCR products from the 11-min nanoPCR and a 2-h conventional benchtop thermocycler, for N1, N2 and RPP30 genes. All of the bands were detected identically for both methods. NTC, non-target control. c, MFS for in situ detection of amplicons. Brightfield (BF) and fluorescence (FL) photographs of an assay mixture before and after 3 min of MFS application are shown. $\mathbf{d}$, N1 gene fluorescence signal changes during MFS application $(n=3)$. 50\% signal recovery was achieved at 3 min. e, Detection of N1, N2 and RPP30 genes before ( - ) and after ( + ) MFS application ( $n=5$ for each measurement). f, Evaluation of the LOD of nanoPCR with different amounts of target N1 RNA $(n=3)$. The detection threshold was set at three times the standard deviation of the signal from a blank. The LOD (dashed horizontal line) was 3.2 copies of gene per $\mu$ l. $\mathbf{g}$, Specificity evaluation for different strains of coronavirus (that is, N1 and N2 genes from SARS-CoV-2, SARS-CoV and Middle East respiratory syndrome coronavirus (MERS-CoV)) ( $n=3$ for each measurement). Data in $\mathbf{d}-\mathbf{g}$ are mean \pm s.d.

for $F_{N 2}$, that maximized the sum of sensitivity and specificity. When these cut-offs were applied to a separate validation set (25 patients with COVID-19 and 25 controls), nanoPCR maintained its high diagnostic power (Fig. 5f). Overall, rapid nanoPCR diagnostics correctly classified all of the clinical samples $(n=150)$ tested (Fig. $5 \mathrm{~g}$ and Supplementary Fig. 14).
Overall, nanoPCR has the potential to decentralize COVID-19 molecular diagnosis. It has practical advantages: (1) the assay is based on well-established RT-PCR to produce more reliable and accurate $(>99 \%)$ results than isothermal amplification tests ${ }^{17,18}$; and (2) nanoPCR considerably shortens the PCR reaction time to enable on-site diagnostics. With these advantages, nanoPCR could 

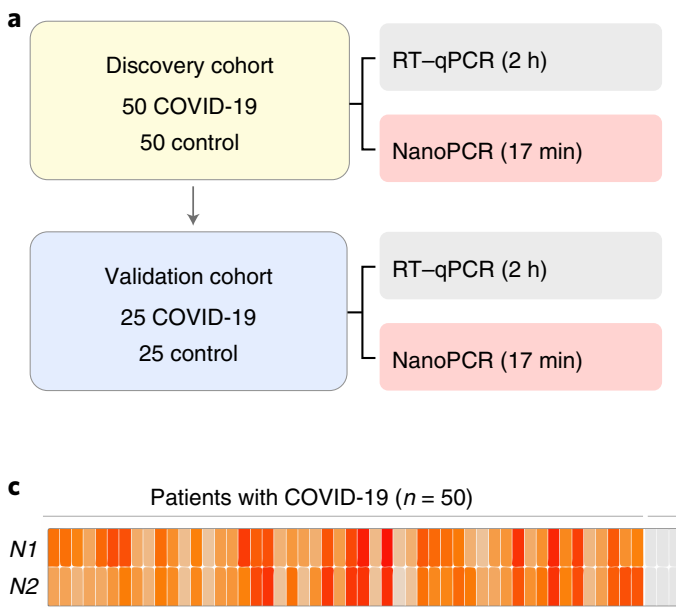

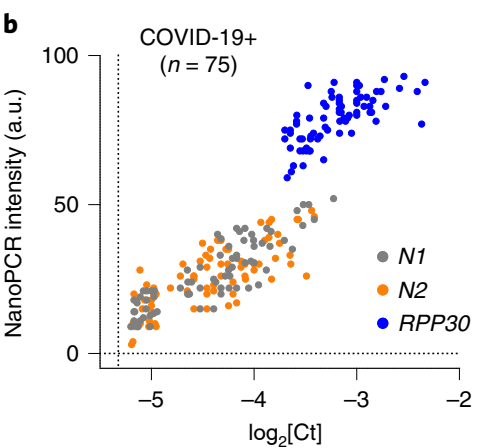

Control individuals without COVID-19 $(n=50)$

Normalized signal, $F$ (a.u.)

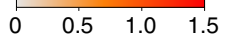

d
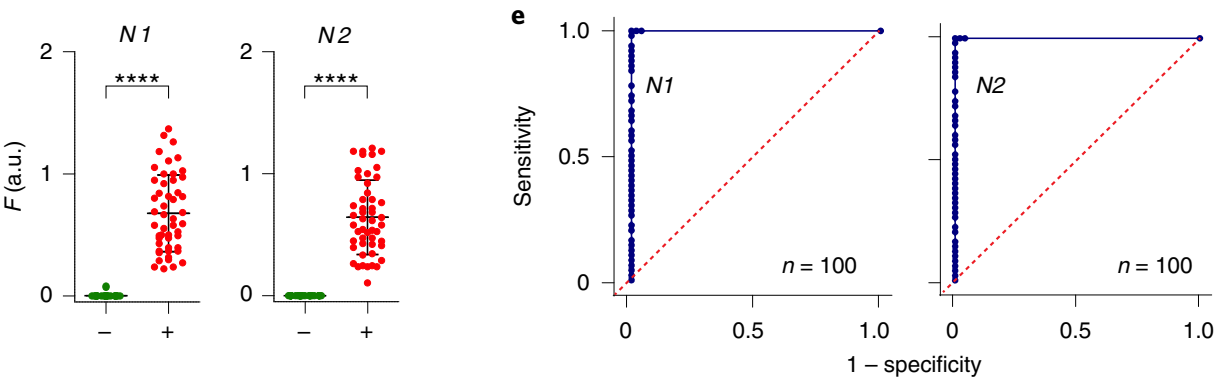

f

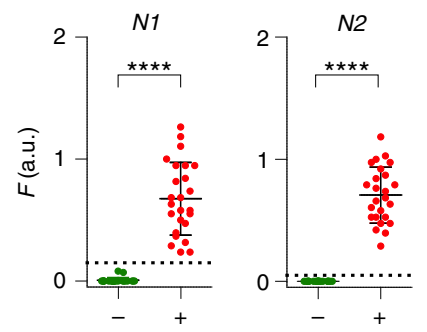

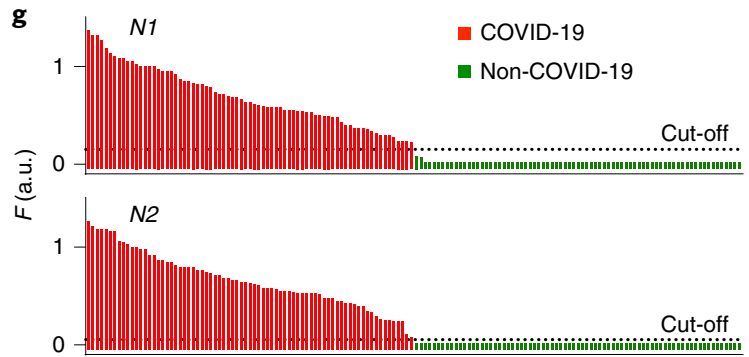

Fig. 5 | Testing of clinical samples with nanoPCR for COVID-19 diagnosis. a, Clinical study design. A total of 150 samples were analysed by nanoPCR and conventional RT-PCR. The first 100 samples were used as a discovery cohort and the remaining 50 were used as a validation cohort. $\mathbf{b}$, Evaluation of analytical concordance between nanoPCR and RT-qPCR. The results were positively correlated (Pearson's $r v a l u e s ; r_{N 1}=0.87 ; r_{N 2}=0.78 ; r_{R P P 30}=0.70$ ). The limit of quantification of both RT-qPCR and nanoPCR was plotted on each axis (broken lines parallel to the axes). Raw intensities from nanoPCR were used. c-e, Analysis of the discovery cohort. c, Control-normalized N1 and N2 signal levels $\left(F_{N 1}\right.$ and $\left.F_{N 2}\right)$ for 50 patients with COVID-19 and 50 controls. d, $F$ values were significantly higher in the patients with COVID-19 (+) than in controls ( $)$ ( ${ }^{\star \star \star \star} P<0.0001$; two-sided $t$-test; $\left.n=100\right)$. e, ROC curves. The AUC was 1 for both $N 1$ and N2. The $F$ cut-off values for diagnostics were determined from the ROC curves: $0.15\left(F_{N 1}\right)$ and $0.05\left(F_{N 2}\right)$. f, The diagnostic accuracy was further confirmed with the validation cohort $(n=50)$. Cut-offs (dotted lines) from the discovery set were applied. As in $\mathbf{d}, F$ values were significantly higher in the patients with COVID-19 $(+)$ than in controls $(-)\left(^{\star \star \star \star} \mathrm{P}<0.0001\right.$; two-sided $t$-test). $\mathbf{g}$, Waterfall distribution of $\mathrm{F}_{\mathrm{N} 1}$ and $\mathrm{F}_{\mathrm{N} 2}$ for all of the samples tested $(n=150)$.

contribute to the decentralization of COVID-19 tests into mobile, ambulatory clinics, mitigating the logistic burden of sample transports. Our prototype system demonstrated such potential, meeting some of the criteria (for example, a sensitivity of $>80 \%$, a specificity of $>97 \%$ and an assay time of $<40 \mathrm{~min}$ ) set by the World Healthcare Organization $^{43}$. Also, MPN synthesis can be optimized for mass production where a single batch currently provides $\sim 30,000$ PCR reactions $(\sim 50 \mathrm{mg})$. For nanoPCR to be used in clinical settings, we envision further technical developments: (1) establishing MPN quality control and good manufacturing practice for mass production; (2) redesigning fluidic cartridges to minimize hands-on times in RNA extraction ${ }^{44}$; and (3) exploring new assay methods, such as heat-inactivated lysis ${ }^{45}$, to offer sample-in and answer-out tests. These efforts will expand nanoPCR's utility in remote or resource-limited settings ${ }^{46,47}$. NanoPCR could also be applied for the prompt diagnosis of other infections, such as acquired immunodeficiency syndrome, tuberculosis and hepatitis ${ }^{48-50}$.

\section{Methods}

Synthesis of MPNs. The MPNs were synthesized as previously described ${ }^{37}$. Briefly, the magnetic core $(\mathrm{M})$ was synthesized by nonhydrolytic thermal decomposition of $\mathrm{Fe}(\mathrm{III})$ acetylacetonate and zinc chloride in oleic acid, oleylamine and trioctylamine at $330^{\circ} \mathrm{C}$. After washing the products with ethanol, $1 \mathrm{mg}$ silica-coated magnetic core with an amine functional group $\left(\mathrm{M} @ \mathrm{SiO}_{2}-\mathrm{NH}_{2}\right)$ was prepared by the sol-gel process of tetraethylorthosilicate and aminopropyltrimethoxysilane. The 
2-nm colloidal Au nanoseeds were mixed with $\mathrm{M} @ \mathrm{SiO}_{2}-\mathrm{NH}_{2}$ to obtain an $\mathrm{Au}$ seed-coated magnetic core $(\mathrm{M} @ \mathrm{Au}=2 \mathrm{~nm})$ at room temperature for $5 \mathrm{~h}$. To prepare the complete Au shell, the Au seeds were grown in 4.81 of titrated Au precursors with $17.2 \mathrm{mg}$ hydroxylamine hydrochloride $\left(\mathrm{NH}_{2} \mathrm{OH}\right)$ for $3 \mathrm{~d}$. After separation with centrifugation and a magnetic column, typically the yield was higher than $65 \%$, corresponding to 150 pmoles $(\sim 50 \mathrm{mg})$. The products were dispersed in $1 \mathrm{mg} \mathrm{ml}^{-1}$ bis( $p$-sulfonatophenyl)phenylphosphine dehydrate dipotassium salt solution for long-term storage.

\section{Transmission electron microscopy (TEM) analysis and EDS mapping.} TEM observations were made using an Atomic Resolution Analytical Electron Microscope (JEM-ARM200F; JEOL) at an acceleration voltage of $200 \mathrm{kV}$. MPNs were freshly prepared on a plasma-cleaned TEM grid (Ultra-Thin PELCO Grids for TEM; Ted Pella). Elemental analysis derived from EDS was performed under atomic resolution mapping followed by visualized indexing.

Plasmonic simulation of MPNs. Electrical fields of MPNs and Au nanoparticles were simulated using the MNPBEM Toolbox in MATLAB (MathWorks) ${ }^{50}$. The toolbox adopted the boundary element method approach ${ }^{39}$, solving Maxwell's equations in metallic nanoparticles wherein homogeneous and isotropic dielectric functions are separated by abrupt interfaces. The simulation assumed that a particle was immersed in water. Wavelength-dependent dielectric functions for $\mathrm{Au}$ and magnetite were used.

Spectral analysis of MPNs. Diluted down to an optical density of 0.1 at $\lambda=535 \mathrm{~nm}$, the aqueous solution of MPNs was subjected to ultraviolet light-aligned spectral analysis using an ultraviolet-visible spectrometer (V-760; Jasco) for absorbance analysis. To obtain scattering measurements for coefficient calculation, the solution was transferred into the integrating sphere (Quanta-phi; HORIBA Scientific) and analysed by photoluminescence spectroscopy (HORIBA Scientific).

Vibrating sample magnetometer measurements. An aqueous solution of MPNs was subject to measurement of magnetization using a vibrating sample magnetometer (VIBRATION 7407-S; Lake Shore Cryotronics). For mass magnetization, the mass of $\mathrm{Fe}, \mathrm{Zn}$ and $\mathrm{Au}$ of each sample was determined using inductively coupled plasma mass spectrometry (7900 ICP-MS; Agilent Technologies)

DLS measurements. The hydrodynamic size of MPNs was measured using a DLS device (Nano ZS90; Malvern) with the following parameters: refractive index $=0.21$ and absorption $=3.32$.

Preparation of nanoPCR mix. NanoPCR mix (total volume $=10 \mu \mathrm{l}$ ) consisting of $0.2 \mu \mathrm{M}$ specific primers (Bioneer; see Supplementary Table 3), $20 \mu \mathrm{M}$ oligo dT, MPNs at an optical density of 4, $200 \mu \mathrm{M}$ dNTPs (N0447S; New England Biolabs), $10 \mu \mathrm{M}$ dithiothreitol, $2 \%$ dimethyl sulfoxide, $0.22 \mu \mathrm{M}$ specific TaqMan probe (Integrated DNA Technologies) (or $1 \times$ SYBR Safe (Invitrogen) in the non-clinical study), $0.5 \mu$ l RNase Inhibitor (LS63; NIPPON Genetics EUROPE) 1 U FastGene Scriptase II (LS63-2; NIPPON Genetics EUROPE), 1 U Hot Start Taq DNA Polymerase (M0495; New England Biolabs), 0.4× Standard Taq Reaction Buffer (containing $10 \mathrm{mM}$ Tris- $\mathrm{HCl}, 50 \mathrm{mM} \mathrm{KCl}$ and $1.5 \mathrm{mM} \mathrm{MgCl}_{2}(\mathrm{pH} 8.3$ ) at $1 \times$ concentration; New England Biolabs) and $0.2 \times$ Reverse Transcription Buffer (containing $10 \mathrm{mM}$ Tris- $\mathrm{HCl}, 50 \mathrm{mM} \mathrm{KCl}$ and $0.1 \%$ Triton $\mathrm{X}-100$ at $1 \times$ concentration (LS63; NIPPON Genetics EUROPE)) was prepared. A certain concentration of synthetic target RNA or viral RNA of clinical samples was applied to the mix, which was then subjected to RT-PCR in the nanoPCR device to automatically give a fluorescence result for the sample.

RNA preparation kit. We engineered a customized RNA-processing kit $\left(20 \times 68 \times 83 \mathrm{~mm}^{3}\right)$ using a three-dimensional (3D) printer (Form 3; Formlabs). Viral RNAs from collected biofluid samples could be purified via the kit's silica gel membrane, where RNAs were bound, washed and eluted. Five individual chambers were used, one at a time, to purify the RNAs. The operational procedure of the kit was as follows: (1) transfer of the sample $(20 \mu \mathrm{l})$ mixed with RNA shield $(20 \mu \mathrm{l})$ onto the top of the membrane (15s); (2) application of viral RNA buffer ( $400 \mu \mathrm{l}$ guanidinium thiocyanate and acid phenol) for capsid degradation (15s); (3) pre-washing and RNA immobilization with ion-exchange chromatography resin ( $1 \mathrm{ml} ; 2 \mathrm{~min})$; (4) application of viral wash buffer ( $500 \mu \mathrm{l}$; containing ethanol) for debris washing (30 s); and (5) application of elution buffer $(15 \mu \mathrm{l})$ and transfer to a tube preloaded with the customized nanoPCR reagents and primers (10 s). All of the reagents and buffers were from a commercial RNA extraction kit (R1034; Zymo Research) and were preloaded into the corresponding chambers before RNA processing. Due to the manufacturing shortcoming for mass production, the disposable RNA preparation kit was used sparingly $(n=5)$ with the sole aim of confirming its capability.

POC device configuration. A 3D-printed plastic housing $\left(150 \times 150 \times 185 \mathrm{~mm}^{3}\right)$ contained optoelectronic components and served as a dark chamber to exclude external light during the RT-PCR and fluorescence measurements. Three sample tubes (PCR-02-C; Corning) were installed separately onto the customized synchronized Ferris wheel. An array of 14 532-nm lasers (CK1872; CivilLaser) was installed for plasmonic heating, and a 310-nm ultraviolet LED (LED310W Thorlabs) was added for fluorescence excitation. The tubes formed a trigonal planar arrangement to make an organized rotation with exact timing, and the bottom solution region of the tube in the reaction order was focused by the lasers (RT-PCR) or the ultraviolet LED (fluorescence detection). The lasers were arranged in a radial formation to enable a common focus on one reaction tube among the three tubes. The ultraviolet LED excited FAM dyes in the sample tube. The fluorescence signal was measured by a detector unit consisting of a bandpass filter (D560; Chroma) and a photodiode (S130C; Thorlabs). The incidence angle was about $55^{\circ}$, which prevented direct illumination onto the detector unit. A stepper motor (17HS3430; MotionKing) rotated the wheel at angles of $120.6^{\circ}$, $120.6^{\circ}$ and $118.8^{\circ}$, and another stepper motor (28BYJ-48; Elegoo) managed the positioning of $52-\mathrm{N}$-grade neodymium magnets $\left(10 \times 10 \times 10 \mathrm{~mm}^{3}\right)$ for MFS. A 3D-printed component holder firmly set the locations of the lasers, the stepper motor and the LED to keep them precisely aligned. A microcontroller board (Arduino Uno R3; Arduino) managed the continuous and sequential operation of the elements, and pulse-width modulation was adopted to control the synchronized actions (Supplementary Fig. 8). The power supply of the nanoPCR device was managed by the PWR button while the nanoPCR procedure was initiated by pushing the PCR button. The fluorescence emission was measured using a sensor read by an optical power console (PM400; Thorlabs). A 12-V Li-ion battery pack supplied the whole system, excluding the console, for over $30 \mathrm{~min}$. All custom-made parts were fabricated using two different 3D printers (3DWOX DP201 (Sindoh) and Form 3 (Formlabs)), considering the manageable resolution and product dimensions

Clinical study. The nasopharyngeal and oropharyngeal specimens collected in the universal transport medium tubes (Asan Pharmaceutical) and sputum specimens were provided by the Chonnam National University Hospital laboratory (Institutional Review Board number CNUH-2020-106) and processed using an automated nucleic acid extraction system (AdvanSure E3 System; LG Chem) to extract viral RNAs. Nasopharyngeal or oropharyngeal swabs contained in universal transport medium were used as provided, whereas sputum samples were diluted with phosphate buffered saline followed by an extraction process. For consistency of the assay, and to avoid any instrumental deviation, one nanoPCR machine was used to assay all of the clinical samples. The results were then compared with the RT-qPCR results, which were also performed using one machine. The analytical measures $(F)$ for diagnostics were defined as $F_{N 1}=I_{N 1} / I_{\mathrm{HK}}$ (for $N 1$ ) and $F_{N 2}=I_{N 2} / I_{\mathrm{HK}}$ (for $N 2$ ), where $I_{N 1}$ and $I_{N 2}$ are the raw intensity values for the $N 1$ and $N 2$ tests, respectively, and $I_{\mathrm{HK}}$ is the fluorescence intensity of a positive control sample. ROC curves were generated using $F_{N 1}$ and $F_{N 2}$. From the discovery cohort, the optimal $F$ cut-offs for COVID-19 diagnostics were determined by choosing values that maximized the sum of sensitivity and specificity: $F_{\mathrm{TH} \_N 1}=0.152$ (for N1) and $F_{\mathrm{TH} \_N 2}=0.053$ (for N2). The analyses were performed using R (version 4.0.2)

RT-qPCR of the clinical specimens. Viral RNA of the clinical samples was added to the RT-PCR mix composed of $0.4 \mu \mathrm{M}$ specific primers (IDT), $200 \mu \mathrm{M}$ dNTP (New England Biolabs), $0.1 \mu \mathrm{M}$ TaqMan probe (IDT), 1.25U Hot Start Taq DNA Polymerase (New England Biolabs), 1.25 U FastGene Scriptase (NIPPON Genetics EUROPE), $1 \times$ Standard Taq Reaction Buffer (New England Biolabs) and Reverse Transcription Buffer (NIPPON Genetics EUROPE). RT-qPCR was then carried out and monitored on a ViiA 7 Real-Time PCR System (Life Technologies) with the following protocol: $94^{\circ} \mathrm{C}$ for $2 \mathrm{~min}$, then 50 cycles of $92^{\circ} \mathrm{C}$ for $8 \mathrm{~s}, 58^{\circ} \mathrm{C}$ for $22 \mathrm{~s}$ and $72^{\circ} \mathrm{C}$ for $40 \mathrm{~s}$. The Ct value was automatically calculated using the system software and was correlated with the result of nanoPCR.

Gel electrophoresis. In $1 \times$ TBE (Tris/Borate/EDTA) running buffer, the PCR product solution was resolved on a $3 \%$ agarose gel containing $1 \times$ SYBR Safe (Invitrogen) as a staining dye at a constant voltage of $120 \mathrm{~V}$ for $40 \mathrm{~min}$. A gel image was taken with a ChemiDoc MP Imaging system (Bio-Rad).

Statistical analysis. All of the data are presented as means \pm s.d. from experiments repeated at least five times unless otherwise specified. Where appropriate, three times the standard deviation, added onto background values, was used as a threshold value for significance.

Reporting Summary. Further information on research design is available in the Nature Research Reporting Summary linked to this article.

\section{Data availability}

The data that support the results of this study are available within the paper and its Supplementary Information files. The raw patient data are available from the authors on reasonable request, subject to approval from the Institutional Review Board of the Chonnam National University Hospital. Non-clinical data generated in this study, including source data and the data used to make the figures, are available in the Supplementary Information files. 
Received: 17 July 2020; Accepted: 1 November 2020;

Published online: 3 December 2020

\section{References}

1. Winter, A. K. \& Hegde, S. T. The important role of serology for COVID-19 control. Lancet Infect. Dis. 20, 758-759 (2020).

2. Cheng, M. P. et al. Diagnostic testing for severe acute respiratory syndrome-related coronavirus 2: a narrative review. Ann. Intern. Med. 172, 726-734 (2020).

3. Weissleder, R., Lee, H., Ko, J. \& Pittet, M. J. COVID-19 diagnostics in context. Sci. Transl. Med. 12, eabc1931 (2020).

4. Wang, Y., Kang, H., Liu, X. \& Tong, Z. Combination of RT-qPCR testing and clinical features for diagnosis of COVID-19 facilitates management of SARS-CoV-2 outbreak. J. Med. Virol. 92, 538-539 (2020).

5. Wang, C. J., Ng, C. Y. \& Brook, R. H. Response to COVID-19 in Taiwan: big data analytics, new technology, and proactive testing. J. Am. Med. Assoc. $\mathbf{3 2 3}$, 1341-1342 (2020).

6. Salathé, M. et al. COVID-19 epidemic in Switzerland: on the importance of testing, contact tracing and isolation. Swiss Med. Wkly 150, w20225 (2020).

7. Kilic, T., Weissleder, R. \& Lee, H. Molecular and immunological diagnostic tests of COVID-19: current status and challenges. iScience 23, 101406 (2020).

8. Ferretti, L. et al. Quantifying SARS-CoV-2 transmission suggests epidemic control with digital contact tracing. Science 368, eabb6936 (2020).

9. Krammer, F. \& Simon, V. Serology assays to manage COVID-19. Science 368, 1060-1061 (2020).

10. Ainsworth, M. et al. Performance characteristics of five immunoassays for SARS-CoV-2: a head-to-head benchmark comparison. Lancet Infect. Dis. https://doi.org/10.1016/S1473-3099(20)30634-4 (2020).

11. Whitman, J. D. et al. Evaluation of SARS-CoV-2 serology assays reveals a range of test performance. Nat. Biotechnol. 38, 1174-1183 (2020).

12. Van Kasteren, P. B. et al. Comparison of commercial RT-PCR diagnostic kits for COVID-19. J. Clin. Virol. 128, 104412 (2020).

13. Jiang, F. et al. Review of the clinical characteristics of coronavirus disease 2019 (COVID-19). J. Gen. Intern. Med. 35, 1545-1549 (2020).

14. Carter, L. J. et al. Assay techniques and test development for COVID-19 diagnosis. ACS Cent. Sci. 6, 591-605 (2020).

15. Petralia, S. \& Conoci, S. PCR technologies for point of care testing: progress and perspectives. ACS Sens. 2, 876-891 (2017).

16. Marx, V. PCR heads into the field. Nat. Methods 12, 393-397 (2015).

17. Mitchell, S. L. \& George, K. S. Evaluation of the COVID19 ID NOW EUA assay. J. Clin. Virol. 128, 104429 (2020).

18. Park, G.-S. et al. Development of reverse transcription loop-mediated isothermal amplification (RT-LAMP) assays targeting SARS-CoV-2. J. Mol. Diagn. 22, 729-735 (2020).

19. You, M. et al. Ultrafast photonic PCR based on photothermal nanomaterials Trends Biotechnol. 38, 637-649 (2020).

20. Son, J. H. et al. Ultrafast photonic PCR. Light Sci. Appl. 4, e280 (2015).

21. Gandolfi, M. et al. Ultrafast thermo-optical dynamics of plasmonic nanoparticles. J. Phys. Chem. C 122, 8655-8666 (2018).

22. Lee, J.-H. et al. Plasmonic photothermal gold bipyramid nanoreactors for ultrafast real-time bioassays. J. Am. Chem. Soc. 139, 8054-8057 (2017).

23. Donner, J. S., Morales-Dalmau, J., Alda, I., Marty, R. \& Quidant, R. Fast and transparent adaptive lens based on plasmonic heating. ACS Photonics 2, 355-360 (2015).

24. Brolo, A. G. Plasmonics for future biosensors. Nat. Photonics 6 709-713 (2012).

25. Kumar, A., Kim, S. \& Nam, J.-M. Plasmonically engineered nanoprobes for biomedical applications. J. Am. Chem. Soc. 138, 14509-14525 (2016).

26. Brongersma, M. L., Halas, N. J. \& Nordlander, P. Plasmon-induced hot carrier science and technology. Nat. Nanotechnol. 10, 25-34 (2015).

27. Ndukaife, J. C., Shalaev, V. M. \& Boltasseva, A. Plasmonics-turning loss into gain. Science 351, 334-335 (2016).

28. Bae, K. et al. Flexible thin-film black gold membranes with ultrabroadband plasmonic nanofocusing for efficient solar vapour generation. Nat. Commun 6, 10103 (2015).

29. Hogan, N. J. et al. Nanoparticles heat through light localization. Nano Lett. 14, 4640-4645 (2014).

30. Son, J. H. et al. Rapid optical cavity PCR. Adv. Healthc. Mater. 5, 167-174 (2016).

31. Lee, Y. et al. Nanoplasmonic on-chip PCR for rapid precision molecular diagnostics. ACS Appl. Mater. Interfaces 12, 12533-12540 (2020).

32. Vanzha, E. et al. Gold nanoparticle-assisted polymerase chain reaction: effects of surface ligands, nanoparticle shape and material. RSC Adv. 6 110146-110154 (2016).

33. Kim, J., Kim, H., Park, J. H. \& Jon, S. Gold nanorod-based photo-PCR system for one-step, rapid detection of bacteria. Nanotheranostics $\mathbf{1}$, $178-185$ (2017).
34. Swierczewska, M., Lee, S. \& Chen, X. The design and application of fluorophore-gold nanoparticle activatable probes. Phys. Chem. Chem. Phys. 13, 9929-9941 (2011).

35. Morozov, V. N., Kolyvanova, M. A., Dement'eva, O. V., Rudoy, V. M. \& Kuzmin, V. A. Fluorescence superquenching of SYBR Green I in crowded DNA by gold nanoparticles. J. Lumin. 219, 116898 (2020).

36. Huang, C. et al. Clinical features of patients infected with 2019 novel coronavirus in Wuhan, China. Lancet 395, 497-506 (2020).

37. Kim, J.-w et al. Single-cell mechanogenetics using monovalent magnetoplasmonic nanoparticles. Nat. Protoc. 12, 1871-1889 (2017).

38. Baffou, G. et al. Photoinduced heating of nanoparticle arrays. ACS Nano 7, 6478-6488 (2013).

39. De Abajo, F. G. \& Howie, A. Retarded field calculation of electron energy loss in inhomogeneous dielectrics. Phys. Rev. B 65, 115418 (2002).

40. Jain, P. K. \& El-Sayed, M. A. Plasmonic coupling in noble metal nanostructures. Chem. Phys. Lett. 487, 153-164 (2010).

41. Kralik, P. \& Ricchi, M. A basic guide to real time PCR in microbial diagnostics: definitions, parameters, and everything. Front. Microbiol. 8 , 108 (2017).

42. Forootan, A. et al. Methods to determine limit of detection and limit of quantification in quantitative real-time PCR (qPCR). Biomol. Detect. Quantif. 12, 1-6 (2017)

43. COVID-19 Target Product Profiles for Priority Diagnostics to Support Response to the COVID-19 Pandemic v.1.0 R\&D Blueprint (World Health Organization, 2020); https://www.who.int/publications/m/item/covid-19-target-product-prof iles-for-priority-diagnostics-to-support-response-to-the-covid19-pandemic-v.0.1

44. Smyrlaki, I. et al. Massive and rapid COVID-19 testing is feasible by extraction-free SARS-CoV-2 RT-PCR. Nature Comm. 11, 4812 (2020)

45. Roda, A. et al. Smartphone-based biosensors: a critical review and perspectives. Trends Analyt. Chem. 79, 317-325 (2016).

46. Liu, D. et al. Trends in miniaturized biosensors for point-of-care testing. Trends Analyt. Chem. 122, 115701 (2020).

47. Van Schalkwyk, C., Maritz, J., Van Zyl, G. U., Preiser, W. \& Welte, A. Pooled PCR testing of dried blood spots for infant HIV diagnosis is cost efficient and accurate. BMC Infect. Dis. 19, 136 (2019).

48. Devonshire, A. S. et al. The use of digital PCR to improve the application of quantitative molecular diagnostic methods for tuberculosis. BMC Infect. Dis. 16, 366 (2016).

49. Probert, W. S. \& Hacker, J. K. New subgenotyping and consensus real-time reverse transcription-PCR assays for hepatitis A outbreak surveillance. J. Clin. Microbiol. 57, e00500-e00519 (2019)

50. Waxenegger, J., Trügler, A. \& Hohenester, U. Plasmonics simulations with the MNPBEM toolbox: consideration of substrates and layer structures. Comput. Phys. Commun. 193, 138-150 (2015).

\section{Acknowledgements}

We thank S.-Y. Cheon for graphic designs and illustrations of figures and POC devices. This work was supported by the Institute for Basic Science (IBS-R026-D1). H.L. was supported in part by US NIH grants R01CA229777, R21DA049577 and U01CA233360, US DOD-W81XWH1910199 and DOD-W81XWH1910194 and the MGH Scholar Fund.

\section{Author contributions}

J. Cheong, H.Y., J.-H.L., H.L. and J. Cheon conceived of and designed the project. J. Cheong and J.-U.L. synthesized the MPNs and performed the material characterizations. H.-J.C. provided the clinical sample. J. Cheong and C.Y.L. conducted the PCR experiments. J. Cheong and H.Y. worked on the nanoPCR design and manufacturing. J. Cheong, H.Y., J.-H.L., H.L. and J. Cheon wrote the manuscript. All authors discussed the results and commented on the manuscript.

\section{Competing interests}

The authors declare no competing interests.

\section{Additional information}

Supplementary information is available for this paper at https://doi.org/10.1038/ s41551-020-00654-0.

Correspondence and requests for materials should be addressed to J.-H.L., H.L. or J.C.

Peer review information Nature Biomedical Engineering thanks the anonymous reviewer(s) for their contribution to the peer review of this work.

Reprints and permissions information is available at www.nature.com/reprints. Publisher's note Springer Nature remains neutral with regard to jurisdictional claims in published maps and institutional affiliations.

(c) The Author(s), under exclusive licence to Springer Nature Limited 2020, corrected publication 2020 


\section{Reporting Summary}

Nature Research wishes to improve the reproducibility of the work that we publish. This form provides structure for consistency and transparency in reporting. For further information on Nature Research policies, see our Editorial Policies and the Editorial Policy Checklist.

\section{Statistics}

For all statistical analyses, confirm that the following items are present in the figure legend, table legend, main text, or Methods section.

n/a Confirmed

$\bigotimes$ The exact sample size $(n)$ for each experimental group/condition, given as a discrete number and unit of measurement

$\bigotimes$ A statement on whether measurements were taken from distinct samples or whether the same sample was measured repeatedly

$\chi$ The statistical test(s) used AND whether they are one- or two-sided

Only common tests should be described solely by name; describe more complex techniques in the Methods section.

$\bigotimes \square$ A description of all covariates tested

Х $\square$ A description of any assumptions or corrections, such as tests of normality and adjustment for multiple comparisons

$\square$ A full description of the statistical parameters including central tendency (e.g. means) or other basic estimates (e.g. regression coefficient)

A full description of the statistical parameters including central tendency (e.g. means) or other basic estima
AND variation (e.g. standard deviation) or associated estimates of uncertainty (e.g. confidence intervals)

$\square$ For null hypothesis testing, the test statistic (e.g. $F, t, r$ ) with confidence intervals, effect sizes, degrees of freedom and $P$ value noted

Give $P$ values as exact values whenever suitable.

Х $\square$ For Bayesian analysis, information on the choice of priors and Markov chain Monte Carlo settings

X $\square$ For hierarchical and complex designs, identification of the appropriate level for tests and full reporting of outcomes

$\square$ Estimates of effect sizes (e.g. Cohen's $d$, Pearson's $r$ ), indicating how they were calculated

Our web collection on statistics for biologists contains articles on many of the points above.

\section{Software and code}

Policy information about availability of computer code

Data collection All data were collected via commercial software (PL, Horiba PL FlourEssence v3.8; TEM, DigitalMicrograph 3.22.1461.0; Temperature, Luxtron fluoroptics v.2.0; VSM, Lake Shore VSM Software 4.9.0; DLS, Zetasizer Software 7.12; RT-PCR, ViiA Applied Biosystems Life science software QuantStudio v1.1; Fluorescent, Thorlab PM400).

Data analysis All data were analysed via commercial or open-source software (PL, Horiba PL FlourEssence v3.8; IRcam, Fluorescent, DNA gel, ImageJ ins.2018; TEM, DigitalMicrograph 3.22.1461.0; Temperature, Luxtron fluoroptics II; VSM, Lake Shore VSM Software 4.9.0; DLS, Zetasizer Software 7.12; RT-PCR, ViiA Applied Biosystems Life science software QuantStudio v1.1; Fluorescent, Thorlab PM400).

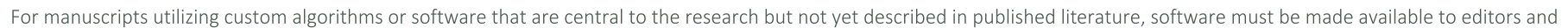

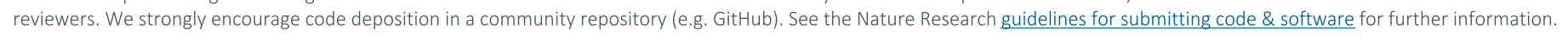

\section{Data}

Policy information about availability of data

All manuscripts must include a data availability statement. This statement should provide the following information, where applicable:

- Accession codes, unique identifiers, or web links for publicly available datasets

- A list of figures that have associated raw data

- A description of any restrictions on data availability

The data supporting the results in this study are available within the paper and its Supplementary Information. The raw patient data are available from the authors on reasonable request, subject to approval from the Institutional Review Board of the Chonnam National University Hospital. Non-clinical data generated in this study, including source data and the data used to make the figures, are available as Supplementary Information. 


\section{Field-specific reporting}

Please select the one below that is the best fit for your research. If you are not sure, read the appropriate sections before making your selection. $\bigotimes$ Life sciences Behavioural \& social sciences Ecological, evolutionary \& environmental sciences

\section{Life sciences study design}

All studies must disclose on these points even when the disclosure is negative.

Sample size $\begin{aligned} & \text { We used } 150 \text { clinical samples (75 COVID-19 positive and } 75 \text { COVID-19 negative). Our clinical study was conducted to test the feasibility of the } \\ & \text { developed assay, and we collected samples that became available on a rolling basis. }\end{aligned}$
Data exclusions
Replication data were excluded from the analyses.
Randomization
$\begin{aligned} & \text { Randomization was not relevant to the study, because the main purpose of the study was to test the performance of the nanoPCR system } \\ & \text { against RT-PCR. }\end{aligned}$
Blinding
$\begin{aligned} & \text { The investigator was not blinded to group allocation during the experiment. Blinding was not relevant because the main purpose of the study } \\ & \text { was to test the performance of the nanoPCR system. The required assay time and sensitivity were compared to the gold standard of COVID-19 } \\ & \text { diagnostics (RT-PCR). }\end{aligned}$

\section{Reporting for specific materials, systems and methods}

We require information from authors about some types of materials, experimental systems and methods used in many studies. Here, indicate whether each material, system or method listed is relevant to your study. If you are not sure if a list item applies to your research, read the appropriate section before selecting a response.

\begin{tabular}{l|l} 
Materials \& experimental systems \\
\hline $\mathrm{n} / \mathrm{a}$ & Involved in the study \\
\hline & $\square$ Antibodies \\
\hline & $\square$ Eukaryotic cell lines \\
$\square$ & $\square$ Palaeontology and archaeology \\
$\square$ & $\square$ Humals and other organisms \\
$\searrow$ & $\square$ Clinical data \\
$\searrow$ & $\square$ Dual use research of concern
\end{tabular}

\begin{tabular}{l|l} 
Methods \\
\hline n/a & Involved in the study \\
$\square$ & $\square$ ChIP-seq \\
$\square$ & $\square$ Flow cytometry \\
$\square$ & $\square$ MRI-based neuroimaging
\end{tabular}

\section{Human research participants}

\section{Policy information about studies involving human research participants}

Population characteristics

Recruitment

Ethics oversight
All samples were from a single institution, Chonnam National University Hospital, Korea. Age and sex were not controlled for. Detailed information about the samples is provided in Table 2 in the Supplementary Information.

Clinicians recruited subjects who presented with COVID-19-related symptoms or who were suspected of SARS-CoV-2 infection.

The study was approved by the Institutional Review Board (IRB) of Chonnam National University Hospital, Korea. 